\title{
Multi-scale 3D mapping of Tomography Data
}

\author{
D. Jha ${ }^{1}$, H. O. Sørensen ${ }^{1}$, D. Müter ${ }^{1}$ and S. L. S. Stipp ${ }^{1}$ \\ 1. Nano-Science Center, Department of Chemistry, University of Copenhagen, \\ Universitetsparken 5, 2100 Copenhagen Ø, Denmark
}

Synchrotron based high-resolution tomography reveals fine structures in a porous media, which strongly determines their flow properties as well as mechanics. Measurements at higher resolution limit the field of view. Lower resolution tomograms on the other hand give a wider field of view but highly compromises the contrast of micro/nano structures in the sample. Lack of contrast in the finer details seriously reduces the image quality as well as quantitative exploitation, limiting the accuracy in the retrieved physical properties. To overcome this, the sample has to be measured at different resolutions on different instruments.

We obtained high-resolution tomograms by linearly translating a chalk sample along the conical X-ray beam at beamline ID22, ESRF [1] as shown in Figure 1. Maximum optical resolution for consolidated chalk samples was observed to be around $100 \mathrm{~nm}$, which leaves some of the microstructures undetected. FIB-SEM tomography [2] can supplement this multi-scale imaging by resolving features down below ten nanometers. It is, however, difficult to obtain a geometrically identical sample position at all instances. This motivates the need of a transformation invariant registration.

We register 3D data with $25 \mathrm{~nm}$ voxel size on to the data with $50 \mathrm{~nm}$ voxel size and so forth until 320 $\mathrm{nm}$. To localize the best possible mapping following steps are performed: 1) Compute the 3D Fourier transformation of the 3D data sets; 2) Transform the $x y$ and yz-planes of the Fourier space to log-polar space; 3) Calculate the phase correlation in between the slices in polar space to retrieve the rotation angles; 4) rotate back one of the Cartesian space 3D data sets and calculate phase correlation with the other. Accordingly, obtained correlation coefficients give a translation, scale and rotation $(\theta$ and $\phi)$ invariant mapping [3] of the multi-scale data sets. Figure 2 shows the tomograms at each positions in the setup illustrated in Figure 1. This method creates a hybrid image where high resolution and wide field of view are combined, thus allowing us to predict an interpolation mechanism relating different length scales. Moreover, it is also instrumental to find the particular resolution at which volume parameters [4] as porosity, tortuosity, pore and grain size distribution, connectivity, and effective-porosity are accurately represented.

[1] T. Weitkamp et al. (1999), Developments in X-ray Tomography II of Proceedings SPIE, p. 311-317

[2] E. Principe and P. Sobol (2005), High-Density FIB-SEM Tomography via Real-Time Imaging. Microscopy and Microanalysis, 11 (Suppl. 02), pp 322-323.

[3] B. Srinivasa Reddy, Biswanath N. Chatterji (1996) An FFT-based technique for translation, rotation, and scale-invariant image registration. IEEE Transactions on Image Processing 5(8): 1266-1271

[4] M. Matrecano (2011), PhD Thesis, Porous Media Characterization By Micro-Tomographic Image Processing. 
[5] The authors thank Finn Engstrøm for providing the chalk samples. Funding was provided by Mærsk Oil and Gas A/S and the Danish National Advanced Technology Foundation (P3 Project), the Danish Council for Independent Research (via DANSCATT) and the European Commission (ITN FP7MINSC).

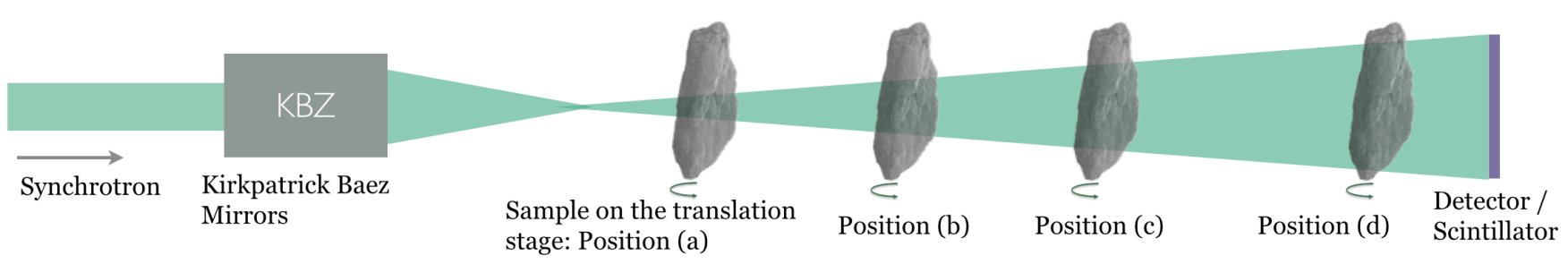

Figure 1. Multi-scale measurement at ID-22 tomography beamline of ESRF. Linear translation along the beamline gives different magnification as a function of distance from the detector.
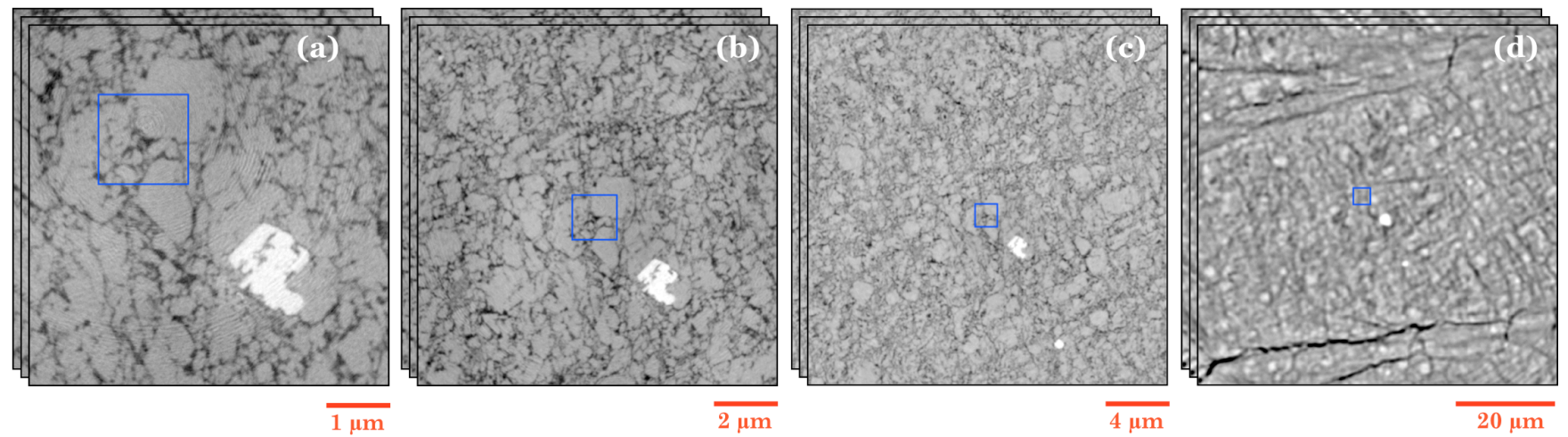

Figure 2. 3D tomograms of chalk obtained at positions (a), (b), (c) and (d) (see Figure 1) have voxel sizes $25,50,100$ and $320 \mathrm{~nm}$ respectively. A 3D sub-volume shown in the blue bounding box is registered at different resolutions tomograms. Tomogram (c) and (d) gives higher field of view but lower resolution unlike (a) and (b). Bright features present in the tomograms are highly absorbing inclusions in chalk. 